\title{
High-fat diet induced changes in gut microbiota and pathogen associate molecular patterns (PAMPS)
}

\author{
L. M. Williams ${ }^{1}$, C. Erridge ${ }^{2}$, C. Grant ${ }^{1}$, A. C. Morris ${ }^{1}$, S. H. Duncan ${ }^{1}$, R. J. Wallace ${ }^{1}$, \\ A. W. Walker ${ }^{3}$ and H. J. Flint ${ }^{1}$ \\ ${ }^{1}$ Rowett Institute of Nutrition and Health, University of Aberdeen, Aberdeen, UK, ${ }^{2}$ Department of Cardiovascular \\ Sciences, University of Leicester, Clinical Sciences Wing, Glenfield General Hospital, Leicester, UK and \\ ${ }^{3}$ Wellcome Trust Sanger Institute, Cambridge, UK
}

A high-fat diet in mice rapidly results in obesity and insulin insensitivity. To further investigate the mechanisms underlying this induction we fed 12 week old male C57B1/6J mice a low- or high-fat (LF or HF) diet: 10\% or 60\% (Kcal) from fat (D12450B and D12492 respectively, Research Diets, USA) for 1 or 12 weeks. Body composition was measured by MRI and glucose tolerance was measured by intraperitioneal glucose tolerance tests (IPGTT). Total numbers of gut bacteria and selected groups were estimated in caecal and faecal contents by fluorescence in situ hybridisation (FISH) microscopy following hybridisation of samples using a panel of probes that target $16 \mathrm{~S}$ rRNA. The relative biological activities of TLR-stimulants present in mouse faeces were measured using a recently developed quantitative bioassay ${ }^{(1,2)}$, employing the measurement of NF- $\kappa$ B-dependent reporter activation in TLR-deficient HEK-293 cells transfected with either TLR2, TLR4/MD2 or TLR5.

Adiposity increased in a linear fashion and glucose tolerance was compromised to a similar extent at both times tested on the HF diet. The HF diet also resulted in more than 2-fold reduction in faecal bacterial numbers at 1 and 12 weeks. Bifidobacteria in HF fed mice were below the level of detection $\left(10^{6} / \mathrm{ml}\right)$ in all but one animal at both 1 and 12 weeks compared to the LF diet where they comprised between 15-17\% of caecal and faecal bacteria. Faecal samples collected after 12 weeks on a HF diet showed an increased proportion of Bacteroidetes and a reduced percentage of Erysipelotrichaceae, based on16S rRNA gene sequencing. Faecal TLR2 and TLR4 activity was found to increase with time $(p<0.001)$ but showed no changes with diet. However, TLR5 activity showed a decrease with HF diet $(p<0.001)$ and no change with time.

These results illustrate that changes in gut bacteria with HF diet in mice are rapid, within one week, and may be due to the replacement of carbohydrate or toxicity of the lipids in the diet. Studies have proposed that bifidobacteria may play a role in reversing or limiting the consequences of a HF diet on inflammation ${ }^{(3)}$ and their loss may explain the increase in inflammation seen on a HF diet. However, we also demonstrate that while TLR2 and TLR4 activity in the colon is not increased on a HF diet TLR5 activity is decreased. This may indicate an alternative mechanism whereby a HF diet is pro-inflammatory, since global TLR 5 deficiency results in impaired epithelial barrier function, chronic PAMP translocation, systemic inflammation and induction of the metabolic syndrome in mice ${ }^{(4)}$.

This work was funded by the Scottish Government Rural and Environment Science and Analytical Services (RESAS).

1. Erridge C, Duncan SH, Bereswill S, et al. (2010) PLoS One 5, e9125.

2. Erridge CJ (2010) Leukoc Biol. 87, 989-99.

3. Cani PD, Neyrinck AM, Fava F et al. (2007) Diabetologia. 50, 2374-83.

4. Vijay-Kumar M, Aitken JD, Carvalho FA et al. (2010) Science 328, 228-31. 